\title{
Journal title change
}

(C) Higher Education Press and Springer-Verlag Berlin Heidelberg 2012

Frontiers of Electrical and Electronic Engineering (FEEE), which was launched in 2006, seeks to provide a multidisciplinary forum for a broad blend of peer-reviewed academic papers in order to promote rapid communication and exchange between scientists in China and abroad. This journal is indexed by INSPEC, CSCD, and SCOPUS.

During the past years, FEEE has been devoted to publishing innovations and applications at the forefront of research in all main branches of electrical and electronic engineering that offer theoretical analysis, numerical evidence, and empirical testing. Since 2010, FEEE especially emphasizes papers in newly emerging and multidisciplinary areas that reflect the international trends of research and development, such as machine learning and intelligence science, bioinformatics, and networked systems. The special issues on the above subjects have raised much attention from researchers. However, it is hard for a quarterly publication journal to catch all the subjects rapidly. In order to reflect the forefront of research more efficiently, FEEE will narrow its interest to information sciences that focus on dealing with biological systems with the new journal title as Quantitative Biology.

Quantitative Biology will be launched in 2013. It will be an interdisciplinary journal that focuses on the original research that uses quantitative approaches and technologies to analyze and integrate biological systems, construct and model engineered life systems, and gain a deeper understanding of life sciences. It aims to provide a platform for not only analysis but also integration and construction of biological systems. It will be a quarterly journal seeking to provide an inter- and multi-disciplinary forum for a broad blend of peer-reviewed academic papers in order to promote efficient communication and exchange between scientists in the East and the West. The content of Quantitative Biology will mainly cover the two broad and related areas:

- bioinformatics and computational biology,

which focuses on dealing with information technologies and computational methodologies that can efficiently and accurately manipulate -omics data and transform molecular information into biological knowledge;

- systems and synthetic biology, which focuses on complex interactions in biological systems and the emergent functional properties, and on the design and construction of new biological functions and systems.

It will try to reflect the significant advances in quantitatively investigating and modeling natural as well as engineered life systems at the molecular and higher levels.

We are grateful to all the Editorial Board members, reviewers, authors, and readers for your kind supports to FEEE, and hope you will continue to support the succeeding Quantitative Biology.

Website: http://journal.hep.com.cn/QB

Online submission: http://mc.manuscriptcentral.com/QB

Frontiers of Electrical and Electronic Engineering

Editorial Office 